DOI: 10.17516/1997-1397-2020-13-3-373-382

УДК 514.16

\title{
Commutative Hypercomplex Numbers and the Geometry of Two Sets
}

\author{
Vladimir A. Kyrov* \\ Gorno-Altai State University \\ Gorno-Altaisk, Russian Federation
}

Received 10.03.2020, received in revised form 16.04.2020, accepted 20.05.2020

\begin{abstract}
The main task of the theory of phenomenologically symmetric geometries of two sets is the classification of such geometries. In this paper, by complexing with associative hypercomplex numbers, functions of a pair of points of new geometries are found by the functions of a pair of points of some wellknown phenomenologically symmetric geometries of two sets (FS GDM). The equations of the groups of motions of these geometries are also found. The phenomenological symmetry of these geometries is established, that is, functional relationships are found between the functions of a pair of points for a certain finite number of arbitrary points. In particular, the $s+1$-component functions of a pair of points of the same ranks are determined by single-component functions of a pair of points of the FS of GDM ranks $(n, n)$ and $(n+1, n)$. Finite equations of motion group and equation expressing their phenomenological symmetry are found.
\end{abstract}

Keywords: geometry of two sets, phenomenological symmetry, group symmetry, hyper-complex numbers.

Citation: V.A.Kyrov, Commutative Hypercomplex Numbers and the Geometry of Two Sets, J. Sib. Fed. Univ. Math. Phys., 2020, 13(3), 373-382. DOI: 10.17516/1997-1397-2020-13-3-373-382.

\section{Introduction}

0.1. In the works [1-3] the definition of one-dimensional phenomenologically symmetric geometry of two sets (PS of GTS) of rank $(n+1, m+1)$ is given, which is given by a differentiable non-degenerate function of a pair of points with open and dense in $R^{m} \times R^{n}$ domain:

$$
f: R^{m} \times R^{n} \rightarrow R
$$

The axiom of phenomenological symmetry is fulfilled: the functional relation

$$
\Phi\left(f\left(\mu_{1}, \nu_{1}\right), f\left(\mu_{1}, \nu_{2}\right), \ldots, f\left(\mu_{n+1}, \nu_{m+1}\right)\right)=0,
$$

for an open and dense subset of the sequences $\left\langle\mu_{1}, \mu_{2}, \ldots, \mu_{n}, \mu_{n+1} ; \nu_{1}, \nu_{2}, \ldots, \nu_{m}, \nu_{m+1}\right\rangle$ of length $n+m+2$ from neighborhood $V\left(\left\langle\mu_{1}, \mu_{2}, \ldots, \mu_{n}, \mu_{n+1} ; \nu_{1}, \nu_{2}, \ldots, \nu_{m}, \nu_{m+1}\right\rangle\right) \subset R^{m(n+1)} \times R^{n(m+1)}$. The function $\Phi$ is differentiable and $\operatorname{rang} \Phi=1$. Points from the first set are denoted $\mu, \mu_{1}, \mu_{2} \ldots$, and points from the second set are $\nu, \nu_{1}, \nu_{2} \ldots$

In the coordinates, the function of a pair of points of the PS of GTS of rank $(n+1, m+1)$ is given as

$$
f(\mu, \nu)=f\left(x^{1}(\mu), \ldots, x^{m}(\mu), \xi^{1}(\nu), \ldots, \xi^{n}(\nu)\right),
$$

*kyrovVA@yandex.ru https://orcid.org/0000-0001-5925-7706

(c) Siberian Federal University. All rights reserved 
where $\left(x^{1}(\mu), \ldots, x^{m}(\mu)\right)$ are the coordinates of the point $\mu \in R^{m}$, and $\left(\xi^{1}(\nu), \ldots, \xi^{n}(\nu)\right)$ are the coordinates of the point $\nu \in R^{n}$.

There is a complete classification of one-dimensional PS of GTS [4], according to which there are PS of GTS only of the ranks $(n+1, n+1),(n+2, n+1)$ and $(4,2)$, where $n \geqslant 1$.

PS of GTS rank $(n+1, n+1)$ :

$$
\begin{gathered}
f(\mu, \nu)=x^{1}(\mu) \xi^{1}(\nu)+\cdots+x^{n}(\mu) \xi^{n}(\nu) \\
f(\mu, \nu)=x^{1}(\mu) \xi^{1}(\nu)+\cdots+x^{n-1}(\mu) \xi^{n-1}(\nu)+x^{n}(\mu)+\xi^{n}(\nu),
\end{gathered}
$$

where $n \geqslant 1$;

PS of GTS rank $(n+2, n+1)$ :

$$
f=f(\mu, \nu)=x^{1}(\mu) \xi^{1}(\nu)+\cdots+x^{n}(\mu) \xi^{n}(\nu)+\xi^{n+1}(\nu),
$$

where $n \geqslant 1$;

PS of GTS $(4,2)$ :

$$
f=f(\mu, \nu)=\frac{x^{1}(\mu) \xi^{1}(\nu)+\xi^{2}(\nu)}{x^{1}(\mu)+\xi^{3}(\nu)} .
$$

0.2. As above, one can define a $s$-metric PS of GTS of rank $(n+1, m+1)$, which is given by a differentiable non-degenerate function of a pair of points with open and dense in $R^{s m} \times R^{s n}$ domain:

$$
f^{\prime}: R^{s m} \times R^{s n} \rightarrow R^{s}
$$

The axiom of phenomenological symmetry is fulfilled: the functional relation

$$
\Phi^{\prime}\left(f^{\prime}\left(\mu_{1}, \nu_{1}\right), f^{\prime}\left(\mu_{1}, \nu_{2}\right), \ldots, f^{\prime}\left(\mu_{n+1}, \nu_{m+1}\right)\right)=0,
$$

for an open and dense subset of the sequences $\left\langle\mu_{1}, \mu_{2}, \ldots, \mu_{n}, \mu_{n+1} ; \nu_{1}, \nu_{2}, \ldots, \nu_{m}, \nu_{m+1}\right\rangle$ of length $n+m+2$ from $V\left(\left\langle\mu_{1}, \mu_{2}, \ldots, \mu_{n}, \mu_{n+1} ; \nu_{1}, \nu_{2}, \ldots, \nu_{m}, \nu_{m+1}\right\rangle\right) \subset R^{s m(n+1)} \times R^{s n(m+1)}$ [3]. The function $\Phi^{\prime}$ is differentiable and $\operatorname{rang} \Phi^{\prime}=s$. There is no complete classification of $s$-metric PS of GTS.

0.3. This work is a continuation of the research published in the article [3]. Here, complexifications of one-dimensional PS of GTS of ranks $(n+1, n+1)$ with $n \geqslant 3$ and PS of GTS of ranks $(n+2, n+1)$ with $n \geqslant 2$ by associative commutative hypercomplex numbers of rank are constructed $s$. For example, hypercomplex numbers of rank 2 are: ordinary complex numbers $\left(i^{2}=-1\right)$, double complex numbers $\left(i^{2}=1\right)$ and dual complex numbers $\left(i^{2}=0\right)[3,5,6]$, associative but noncommutative hypercomplex numbers of rank 4 are quaternions [5]. As a result of complexification, functions of a pair of points of $s$-metric PS of GTS are obtained. This method was tested in [3] and [5].

Note that the cases $n=1$ and $n=2$ for PS of GTS rank $(n+1, n+1)$, as well as the case $n=1$ for PS of GTS rank $(n+2, n+1)$ was previously considered in [3] over the algebra of associative hypercomplex numbers.

\section{Algebra of hypercomplex numbers and matrix algebra over hypercomplex numbers}

1.1. The results of this item are given by article [3]. Consider the real associative commutative algebra $L$ of hypercomplex numbers of order $s$ ( [7], p. 462). An arbitrary hypercomplex number 
has the form: $x=x_{0}+x_{1} i_{1}+\cdots+x_{s-1} i_{s-1}$, where $x_{0}, x_{1}, \ldots, x_{s-1} \in R, i_{0}=1, i_{1}, \ldots, i_{s-1}$ are imaginary units. Addition and multiplication by a real number are component determined, and the product is written so: for arbitrary $x, y \in L$,

$$
x y=\sum_{k, l=0}^{n} x_{k} y_{l} i_{k} i_{l} .
$$

The product of imaginary units $i_{k} i_{l} \in L$ is defined by a special table. Denote by $U(L) \subset L$ the set of invertible elements. The set $U(L)$ is open and dense in $L$ and is a group by multiplication.

1.2. Let $M_{m}$ be the set of matrices of size $m \times m$ over the algebra of associative commutative hypercomplex numbers $L, m>1$. The addition of such matrices and multiplication by a hypercomplex number is determined in the usual way. The product of matrices is also determined by the rule "row by column". One can prove that $M_{m}$ is a linear associative algebra ([7], p. 184).

Consider the matrix $A=\left(a_{i j}\right) \in M_{m}$, where $i, j=1, \ldots, m$. Denote by $|A|$ the determinant of this matrix, and by $A_{i k}$ - the algebraic complement of the element $a_{i k}$. Further we use the well-known statement from linear algebra.

Proposition 1.1 ([8], p. 50). A square matrix $A$ with elements from a commutative ring $K$ with unity has an inverse matrix with elements from $K$ when and only if the determinant of the matrix $A$ is invertible into $K$.

From this statement it follows that over an algebra of associative commutative hypercomplex numbers $L$, the matrix $A$ is invertible if and only if $|A| \in U(L)$. The set of invertible matrices in $M_{m}$ is denoted by $\left.U M_{m}\right)$. This set is open and dense in $M_{m}$. The inverse matrix to the matrix $A \in U\left(M_{m}\right)$ is denoted $A^{-1}=\left(\alpha_{i k}\right)$ and its element $\alpha_{i k}$ is calculated by the formula ([9], p. 26):

$$
\alpha_{i k}=A_{k i}|A|^{-1} \text {. }
$$

\section{Classification of one-metric phenomenologically symmetric geometries of two sets (PS of GTS)}

2.1. In the introduction, the definition of a one-metric PS of GTS of rank $(n+1, m+1)$ is given, and the functions of a pair of points for a PS of GTS of rank $(n+1, n+1)$, of a PS of GTS of rank $(n+1, n)$ and PS of GTS rank $(4,2)$. For them, functional connections are known [1] and [4]:

For PS of GTS rank $(n+1, n+1)$ :

first solution (function of a pair of points (1)):

$$
\left|\begin{array}{cccc}
f\left(\mu_{1}, \nu_{1}\right) & f\left(\mu_{1}, \nu_{2}\right) & \cdots & f\left(\mu_{1}, \nu_{n}\right) \\
f\left(\mu_{2}, \nu_{1}\right) & f\left(\mu_{2}, \nu_{2}\right) & \cdots & f\left(\mu_{2}, \nu_{n}\right) \\
\cdots & \cdots & \cdots & \cdots \\
f\left(\mu_{n}, \nu_{1}\right) & f\left(\mu_{n}, \nu_{2}\right) & \cdots & f\left(\mu_{n}, \nu_{n}\right)
\end{array}\right|=0
$$

second solution (function of a pair of points (2)):

$$
\left|\begin{array}{ccccc}
0 & 1 & 1 & \cdots & 1 \\
1 & f\left(\mu_{1}, \nu_{1}\right) & f\left(\mu_{1}, \nu_{2}\right) & \cdots & f\left(\mu_{1}, \nu_{n}\right) \\
1 & f\left(\mu_{2}, \nu_{1}\right) & f\left(\mu_{2}, \nu_{2}\right) & \cdots & f\left(\mu_{2}, \nu_{n}\right) \\
\cdots & \cdots & \cdots & \cdots & \cdots \\
1 & f\left(\mu_{n}, \nu_{1}\right) & f\left(\mu_{n}, \nu_{2}\right) & \cdots & f\left(\mu_{n}, \nu_{n}\right)
\end{array}\right|=0 .
$$


For PS of GTS rank $(n+1, n)$ :

$$
\left|\begin{array}{ccccc}
f\left(\mu_{1}, \nu_{1}\right) & f\left(\mu_{1}, \nu_{2}\right) & \cdots & f\left(\mu_{1}, \nu_{n-1}\right) & 1 \\
f\left(\mu_{2}, \nu_{1}\right) & f\left(\mu_{2}, \nu_{2}\right) & \cdots & f\left(\mu_{2}, \nu_{n-1}\right) & 1 \\
\cdots & \cdots & \cdots & \cdots & \cdots \\
f\left(\mu_{n}, \nu_{1}\right) & f\left(\mu_{n}, \nu_{2}\right) & \cdots & f\left(\mu_{n}, \nu_{n-1}\right) & 1
\end{array}\right|=0 .
$$

For PS of GTS rank $(4,2)$ :

$$
\left|\begin{array}{llll}
f\left(\mu_{1}, \nu_{1}\right) & f\left(\mu_{1}, \nu_{2}\right) & f\left(\mu_{1}, \nu_{1}\right) f\left(\mu_{1}, \nu_{2}\right) & 1 \\
f\left(\mu_{2}, \nu_{1}\right) & f\left(\mu_{2}, \nu_{2}\right) & f\left(\mu_{2}, \nu_{1}\right) f\left(\mu_{2}, \nu_{2}\right) & 1 \\
f\left(\mu_{3}, \nu_{1}\right) & f\left(\mu_{3}, \nu_{2}\right) & f\left(\mu_{3}, \nu_{1}\right) f\left(\mu_{3}, \nu_{2}\right) & 1 \\
f\left(\mu_{4}, \nu_{1}\right) & f\left(\mu_{4}, \nu_{2}\right) & f\left(\mu_{4}, \nu_{1}\right) f\left(\mu_{4}, \nu_{2}\right) & 1
\end{array}\right|=0 .
$$

2.2. The concept of motion in GTS is introduced as a set of locally diffeomorphic transformations

$$
x^{\prime}=\lambda(x), \quad \xi^{\prime}=\sigma(\xi)
$$

of $R^{m}$ and $R^{n}$ varieties preserving the function pairs of points:

$$
f\left(x^{\prime}, \xi^{\prime}\right)=f(\lambda(x), \quad \sigma(\xi))=f(x, \xi) .
$$

Relation (9) is a functional equation for a group of motions, solving which are the equations of this group:

For PS of GTS rank $(n+1, n+1)$ :

first solution:

$$
X^{\prime}=A X, \quad \Xi^{\prime}=A^{-1} \Xi,
$$

where $X^{\prime}=\left(\begin{array}{c}x^{\prime 1} \\ \cdots \\ x^{\prime n}\end{array}\right), X=\left(\begin{array}{c}x^{1} \\ \cdots \\ x^{n}\end{array}\right), \Xi^{\prime}=\left(\begin{array}{c}\xi^{\prime 1} \\ \cdots \\ \xi^{\prime n}\end{array}\right), \Xi=\left(\begin{array}{c}\xi^{1} \\ \cdots \\ \xi^{n}\end{array}\right), A=\left(\begin{array}{ccc}a^{11} & \cdots & a^{1 n} \\ \cdots & \cdots & \cdots \\ a^{n 1} & \cdots & a^{n n}\end{array}\right)$ is nondegenerate matrix;

second solution:

$$
\begin{gathered}
X^{\prime}=A X+B, \quad x^{\prime n}=x^{n}+C^{T} X+b^{n}, \\
\Xi^{\prime}=A^{-1}(\Xi-C), \quad \xi^{\prime n}=\xi^{n}-B^{T} A^{-1}(\Xi-C)-b^{n},
\end{gathered}
$$

where $X^{\prime}=\left(\begin{array}{c}x^{\prime 1} \\ \ldots \\ x^{\prime n-1}\end{array}\right), \quad X=\left(\begin{array}{c}x^{1} \\ \ldots \\ x^{n-1}\end{array}\right), \quad \Xi^{\prime}=\left(\begin{array}{c}\xi^{\prime 1} \\ \ldots \\ \xi^{\prime n-1}\end{array}\right), \quad \Xi=\left(\begin{array}{c}\xi^{1} \\ \ldots \\ \xi^{n-1}\end{array}\right), \quad B=\left(\begin{array}{c}b^{1} \\ \ldots \\ b^{n-1}\end{array}\right)$, $C=\left(\begin{array}{c}c^{1} \\ \cdots \\ c^{n-1}\end{array}\right), A=\left(\begin{array}{ccc}a^{11} & \cdots & a^{1(n-1)} \\ \cdots & \cdots & \cdots \\ a^{(n-1) 1} & \cdots & a^{(n-1)(n-1)}\end{array}\right)$ nondegenerate matrix.

For PS of GTS rank $(n+1, n)$ :

$$
X^{\prime}=A X+B, \quad \Xi^{\prime}=A^{-1} \Xi, \quad \xi^{\prime n}=\xi^{n}-B^{T} A^{-1} \Xi,
$$

where $X^{\prime}=\left(\begin{array}{c}x^{\prime 1} \\ \ldots \\ x^{\prime n-1}\end{array}\right), \quad X=\left(\begin{array}{c}x^{1} \\ \ldots \\ x^{n-1}\end{array}\right), \quad \Xi^{\prime}=\left(\begin{array}{c}\xi^{\prime 1} \\ \ldots \\ \xi^{\prime n-1}\end{array}\right), \quad \Xi=\left(\begin{array}{c}\xi^{1} \\ \ldots \\ \xi^{n-1}\end{array}\right), \quad B=\left(\begin{array}{c}b^{1} \\ \ldots \\ b^{n-1}\end{array}\right)$, $A=\left(\begin{array}{ccc}a^{11} & \cdots & a^{1(n-1)} \\ \cdots & \cdots & \cdots \\ a^{(n-1) 1} & \cdots & a^{(n-1)(n-1)}\end{array}\right)$ nondegenerate matrix. 
For PS of GTS rank $(4,2)$ :

$$
\begin{aligned}
& x^{1 \prime}=\left(a x^{1}+b\right) /\left(c x^{1}+d\right), \quad \xi^{1 \prime}=\left(d \xi^{1}-c \xi^{2}\right) /\left(d-c \xi^{3}\right), \\
& \xi^{2 \prime}=\left(a \xi^{2}-b \xi^{1}\right) /\left(d-c \xi^{3}\right), \quad \xi^{3 \prime}=\left(a \xi^{3}-b\right) /\left(d-c \xi^{3}\right),
\end{aligned}
$$

where $a d-b c=1$.

It should be noted that group and phenomenological symmetries for PS of GTS are equivalent in the following sense: by the function of a pair of points, you can find a group of motions, and by a group of motions - a function of a pair of points $[1,2,4]$.

\section{Complexification of one-metric PS of GTS rank $(n+1, n+1)$ hypercomplex numbers}

3.1. Consider a one-metric FS of GDM rank $(n+1, n+1)$, which exists in two variants ( [1], p. 63), defined by the functions of a pair of points (1) and (2) in $R^{n} \times R^{n}$. For $n \geqslant 2$ these options are not equivalent. For the first solution, the functional relationship is expressed by equation (5).

The group symmetry of degree $n^{2}$ is determined by the $n^{2}$-parametric group of motions with equations (10) for the function of the pair of points (1), which satisfies the identity (9).

3.2. We carry out the complexification of the function of the pair of points (1), passing to the corresponding hypercomplex functions and coordinates, assuming

$$
f=\sum_{k=1}^{s} f_{k} i_{k}, \quad x=\sum_{k=1}^{s} x_{k} i_{k}, \quad \xi=\sum_{k=1}^{s} \xi_{k} i_{k} .
$$

As a result, we obtain the $s$-component function of a pair of points

$$
f_{\mathrm{K}}=x^{1} \xi^{1}+\cdots+x^{n} \xi^{n},
$$

where $x^{1}, \ldots, x^{n}, \xi^{1}, \ldots, \xi^{n}, f_{k} \in L$. Phenomenological symmetry, as is easily seen, if we use the formula (14), is given by the identity:

$$
\left|\begin{array}{cccc}
f_{\mathrm{K}}\left(\mu_{1}, \nu_{1}\right) & f_{\mathrm{K}}\left(\mu_{1}, \nu_{2}\right) & \cdots & f_{\mathrm{K}}\left(\mu_{1}, \nu_{n}\right) \\
f_{\mathrm{K}}\left(\mu_{2}, \nu_{1}\right) & f_{\mathrm{K}}\left(\mu_{2}, \nu_{2}\right) & \cdots & f_{\mathrm{K}}\left(\mu_{2}, \nu_{n}\right) \\
\cdots & \cdots & \cdots & \cdots \\
f_{\mathrm{K}}\left(\mu_{n}, \nu_{1}\right) & f_{\mathrm{K}}\left(\mu_{n}, \nu_{2}\right) & \cdots & f_{\mathrm{K}}\left(\mu_{n}, \nu_{n}\right)
\end{array}\right|=0 .
$$

3.3. Let us find the group of motions for the complexification PS of GTS rank $(n+1, n+1)$. To do this, we solve the functional equation

$$
x^{\prime 1} \xi^{1}+\cdots+x^{\prime n} \xi^{\prime n}=x^{1} \xi^{1}+\cdots+x^{n} \xi^{n}
$$

on a set of motions.

Theorem 1. The group of motions of the complexification PS of GTS of rank $(n+1, n+1)$ with the function of a pair of points (14) is given by the equations

$$
X^{\prime}=X A, \quad \Xi^{\prime}=A^{-1} \Xi
$$

in which $X=\left(\begin{array}{lll}x^{1} & \cdots & x^{n}\end{array}\right), \Xi=\left(\begin{array}{c}\xi^{1} \\ \cdots \\ \xi^{n}\end{array}\right), A=\left(\begin{array}{ccc}a_{1}^{1} & \cdots & a_{1}^{n} \\ \cdots & \cdots & \cdots \\ a_{n}^{1} & \cdots & a_{n}^{n}\end{array}\right)=$ const $\in U\left(M_{n}\right)$. 
Proof. We write the identity (15) for $n$ pairs of points $\left\langle\mu \nu_{1}\right\rangle, \ldots,\left\langle\mu \nu_{n}\right\rangle$ :

$$
\begin{aligned}
& x^{\prime 1} \xi^{\prime 1}\left(\nu_{1}\right)+\cdots+x^{\prime n} \xi^{\prime n}\left(\nu_{1}\right)=x^{1} \xi^{1}\left(\nu_{1}\right)+\cdots+x^{n} \xi^{n}\left(\nu_{1}\right), \\
& x^{1} \xi^{\prime 1}\left(\nu_{n}\right)+\cdots+x^{\prime n} \xi^{\prime n}\left(\nu_{n}\right)=x^{1} \xi^{1}\left(\nu_{n}\right)+\cdots+x^{n} \xi^{n}\left(\nu_{n}\right) .
\end{aligned}
$$

For convenience, the last equalities are rewritten in a matrix form:

$$
X^{\prime} D^{\prime}=X D
$$

where $D=\left(\begin{array}{ccc}\xi^{1}\left(\nu_{1}\right) & \cdots & \xi^{1}\left(\nu_{n}\right) \\ \cdots & \cdots & \cdots \\ \xi^{n}\left(\nu_{1}\right) & \cdots & \xi^{n}\left(\nu_{n}\right)\end{array}\right) \in U\left(M_{n}\right)$. Resolving, we have

$$
X^{\prime}=X D D^{\prime-1}
$$

It can be seen that the variables are divided. Therefore $A=D D^{\prime-1}=\left(\begin{array}{ccc}a_{1}^{1} & \cdots & a_{1}^{n} \\ \cdots & \cdots & \cdots \\ a_{n}^{1} & \cdots & a_{n}^{n}\end{array}\right)=$ $=$ const $\in U\left(M_{n}\right)$. In this way,

$$
X^{\prime}=X A
$$

Similarly, identity (15) is written for the sequences $\left\langle\mu_{1} \nu\right\rangle, \ldots,\left\langle\mu_{n} \nu\right\rangle$, and then rewritten in a matrix form:

$$
U^{\prime} \Xi^{\prime}=U \Xi
$$

where $U=\left(\begin{array}{ccc}x^{1}\left(\mu_{1}\right) & \cdots & x^{n}\left(\mu_{1}\right) \\ \cdots & \cdots & \cdots \\ x^{1}\left(\mu_{n}\right) & \cdots & x^{n}\left(\mu_{n}\right)\end{array}\right) \in U\left(M_{n}\right), U^{\prime}=U A$. Then

$$
U A \Xi^{\prime}=U \Xi, \quad \Xi^{\prime}=(U A)^{-1} U \Xi=A^{-1} U^{-1} U \Xi=A^{-1} \Xi .
$$

Thus, we obtain (16).

Obviously, the group of motions (16) is a $s n^{2}$-parametric group, which includes $s n^{2}$ real parameters.

3.4. For the second solution, the functional relationship is expressed by equation (6). The group symmetry of degree $n^{2}$ is determined by the $n^{2}$-parameter group of motions with equations (11) for the function of the pair of points (2), which satisfies the identity (9).

3.5. When passing to hypercomplex coordinates in expression (2), we obtain the $s$-component function of a pair of points

$$
f_{\mathrm{K}}=x^{1} \xi^{1}+\cdots+x^{n-1} \xi^{n-1}+x^{n}+\xi^{n},
$$

where $x^{1}, \ldots, x^{n}, \xi^{1}, \ldots, \xi^{n}, f_{k} \in L$.

Phenomenological symmetry, as is easily seen, if we use the formula (17), is given by the identity:

$$
\left|\begin{array}{ccccc}
0 & 1 & 1 & \cdots & 1 \\
1 & f_{\mathrm{K}}\left(\mu_{1}, \nu_{1}\right) & f_{\mathrm{K}}\left(\mu_{1}, \nu_{2}\right) & \cdots & f_{\mathrm{K}}\left(\mu_{1}, \nu_{n}\right) \\
1 & f_{\mathrm{K}}\left(\mu_{2}, \nu_{1}\right) & f_{\mathrm{K}}\left(\mu_{2}, \nu_{2}\right) & \cdots & f_{\mathrm{K}}\left(\mu_{2}, \nu_{n}\right) \\
\cdots & \cdots & \cdots & \cdots & \cdots \\
1 & f_{\mathrm{K}}\left(\mu_{n}, \nu_{1}\right) & f_{\mathrm{K}}\left(\mu_{n}, \nu_{2}\right) & \cdots & f_{\mathrm{K}}\left(\mu_{n}, \nu_{n}\right)
\end{array}\right|=0 .
$$


3.6. We find the group of motions for the second complexification PS of GTS rank $(n+1, n+1)$. For this, we solve the functional equation

$$
x^{\prime 1} \xi^{\prime 1}+\cdots+x^{\prime n-1} \xi^{\prime n-1}+x^{\prime n}+\xi^{\prime n}=x^{1} \xi^{1}+\cdots+x^{n-1} \xi^{n-1}+x^{n}+\xi^{n}
$$

on a set of movements.

Theorem 2. The group of motions of the complexification second PS of GTS of rank $(n+1, n+1)$ with the function of a pair of points (17) is given by the equations

$$
\begin{gathered}
X^{\prime}=X A+B, \quad x^{\prime n}=x^{n}+X C^{T}+b^{n} ; \\
\Xi^{\prime}=A^{-1}\left(\Xi-C^{T}\right), \quad \xi^{\prime n}=\xi^{n}-B A^{-1}\left(\Xi-C^{T}\right)-b^{n},
\end{gathered}
$$

in which $\Xi=\left(\begin{array}{c}\xi^{1} \\ \ldots \\ \xi^{n-1}\end{array}\right), X=\left(\begin{array}{lll}x^{1} & \cdots & x^{n-1}\end{array}\right), B=\left(\begin{array}{c}b^{1} \\ \ldots \\ b^{n-1}\end{array}\right)=$ const, $C=\left(\begin{array}{c}c^{1} \\ \ldots \\ c^{n-1}\end{array}\right)=$ const, $b^{n}=$ const, $A=\left(\begin{array}{ccc}a_{1}^{1} & \cdots & a_{1}^{n-1} \\ \cdots & \cdots & \cdots \\ a_{n-1}^{1} & \cdots & a_{n-1}^{n-1}\end{array}\right)=$ const $\in U\left(M_{n-1}\right)$.

The proof of this theorem is similar to the proof of Theorem 1; therefore, it is omitted.

It is obvious that the group of motions (18) is a $s n^{2}$-parametric group, which includes $s n^{2}$ real parameters.

\section{Complexification of one-metric PS of GTS rank $(n+1, n)$ hypercomplex numbers}

4.1. We now turn to a one-metric FS of a GDM of rank $(n+1, n),([1]$, p. 63$)$ defined by the function of the pair of points (3) in $R^{n-1} \times R^{n}$. The functional relationship is given by formula (7). The group symmetry of degree $n(n-1) 2$ is determined by the $n(n-1)$-parametric group of motions with equations (12) for the metric function (3), which satisfies the identity (9).

4.2. In the transition to the hypercomplex coordinates in expression (3), we get the $s$-component function of a pair of points

$$
f_{\mathrm{K}}=x^{1} \xi^{1}+\cdots+x^{n-1} \xi^{n-1}+\xi^{n},
$$

where $x^{1}, \ldots, x^{n-1}, \xi^{1}, \ldots, \xi^{n}, f_{\mathrm{K}} \in L$. Phenomenological symmetry, as is easily seen, if we use the formula (19), is given by the identity:

$$
\left|\begin{array}{ccccc}
f_{\mathrm{K}}\left(\mu_{1}, \nu_{1}\right) & f_{\mathrm{K}}\left(\mu_{1}, \nu_{2}\right) & \cdots & f_{\mathrm{K}}\left(\mu_{1}, \nu_{n-1}\right) & 1 \\
f_{\mathrm{K}}\left(\mu_{2}, \nu_{1}\right) & f_{\mathrm{K}}\left(\mu_{2}, \nu_{2}\right) & \cdots & f_{\mathrm{K}}\left(\mu_{2}, \nu_{n-1}\right) & 1 \\
\ldots & \cdots & \cdots & \cdots & \cdots \\
f_{\mathrm{K}}\left(\mu_{n}, \nu_{1}\right) & f_{\mathrm{K}}\left(\mu_{n}, \nu_{2}\right) & \cdots & f_{\mathrm{K}}\left(\mu_{n}, \nu_{n-1}\right) & 1
\end{array}\right|=0 .
$$

4.3. Let us find the group of motions for the complexification PS of GTS rank $(n+1, n)$. For this, we solve the functional equation

$$
x^{\prime 1} \xi^{1}+\cdots+x^{\prime n-1} \xi^{\prime n-1}+\xi^{\prime n}=x^{1} \xi^{1}+\cdots+x^{n-1} \xi^{n-1}+\xi^{n}
$$

on a set of movements. 
Theorem 3. The group of motions of the complexification second PS of GTS of rank $(n+1, n)$ with the function of a pair of points (19) is given by the equations

$$
X^{\prime}=X A+B, \quad \Xi^{\prime}=A^{-1} \Xi, \quad \xi^{\prime n}=\xi^{n}-B A^{-1} \Xi,
$$

in which the notation is entered $\Xi=\left(\begin{array}{c}\xi^{1} \\ \cdots \\ \xi^{n-1}\end{array}\right), X=\left(\begin{array}{lll}x^{1} & \cdots & x^{n-1}\end{array}\right), B=\left(\begin{array}{c}b^{1} \\ \cdots \\ b^{n-1}\end{array}\right)=$ const, $A=\left(\begin{array}{ccc}a_{1}^{1} & \cdots & a_{1}^{n-1} \\ \cdots & \cdots & \cdots \\ a_{n-1}^{1} & \cdots & a_{n-1}^{n-1}\end{array}\right)=$ const $\in U\left(M_{n-1}\right)$.

The proof of this theorem, as well as the previous one, is similar to the proof of Theorem 1; therefore, it is omitted.

It is obvious that the group of motions $(20)$ is a $\operatorname{sn}(n-1)$-parametric group, which includes $s n(n-1)$ real parameters.

\section{Complexification of one-metric PS of GTS rank $(4,2)$ hypercomplex numbers}

5.1. Finally, we turn to a one-metric PS of GTS of rank $(4,2)$, ([1], p. 63) defined by the function of a pair of points (4) in $R \times R^{3}$. The functional relationship is given by formula (8). The group symmetry of degree $n(n-1) 2$ is determined by the $n(n-1)$-parametric group of motions with equations (13) for the metric function (3), which satisfies the identity (9).

5.2. In the transition in expression (4) to hypercomplex coordinates, we obtain the $s$ component function of a pair of points

$$
f_{\mathrm{K}}=\left(x^{1} \xi^{1}+\xi^{2}\right)\left(x^{1}+\xi^{3}\right)^{-1},
$$

where $x^{1}, \xi^{1}, \xi^{2}, \xi^{3}, f_{\mathrm{K}} \in L, x^{1}+\xi^{3} \in U(L)$. Phenomenological symmetry, as is easily seen, if we use the formula (21), is given by the identity:

$$
\left|\begin{array}{llll}
f_{\mathrm{K}}\left(\mu_{1}, \nu_{1}\right) & f_{\mathrm{K}}\left(\mu_{1}, \nu_{2}\right) & f_{\mathrm{K}}\left(\mu_{1}, \nu_{1}\right) f_{\mathrm{K}}\left(\mu_{1}, \nu_{2}\right) & 1 \\
f_{\mathrm{K}}\left(\mu_{2}, \nu_{1}\right) & f_{\mathrm{K}}\left(\mu_{2}, \nu_{2}\right) & f_{\mathrm{K}}\left(\mu_{2}, \nu_{1}\right) f_{\mathrm{K}}\left(\mu_{2}, \nu_{2}\right) & 1 \\
f_{\mathrm{K}}\left(\mu_{3}, \nu_{1}\right) & f_{\mathrm{K}}\left(\mu_{3}, \nu_{2}\right) & f_{\mathrm{K}}\left(\mu_{3}, \nu_{1}\right) f_{\mathrm{K}}\left(\mu_{3}, \nu_{2}\right) & 1 \\
f_{\mathrm{K}}\left(\mu_{4}, \nu_{1}\right) & f_{\mathrm{K}}\left(\mu_{4}, \nu_{2}\right) & f_{\mathrm{K}}\left(\mu_{4}, \nu_{1}\right) f_{\mathrm{K}}\left(\mu_{4}, \nu_{2}\right) & 1
\end{array}\right|=0
$$

5.3. Let us find the group of motions for the complexification PS of GTS rank (4,2). For this, we solve the functional equation

$$
\left(x^{1 \prime} \xi^{1 \prime}+\xi^{2 \prime}\right)\left(x^{1 \prime}+\xi^{3 \prime}\right)^{-1}=\left(x^{1} \xi^{1}+\xi^{2}\right)\left(x^{1}+\xi^{3}\right)^{-1}
$$

on a set of movements.

Theorem 4. The group of motions of the complexification second PS of GTS of rank $(4,2)$ with the function of a pair of points (21) is given by the equations

$$
\begin{cases}x^{1 \prime}=\left(a x^{1}+b\right)\left(c x^{1}+d\right)^{-1}, & \xi^{1 \prime}=\left(d \xi^{1}-c \xi^{2}\right)\left(d-c \xi^{3}\right)^{-1} \\ \xi^{2 \prime}=\left(a \xi^{2}-b \xi^{1}\right)\left(d-c \xi^{3}\right)^{-1}, & \xi^{3 \prime}=\left(a \xi^{3}-b\right)\left(d-c \xi^{3}\right)^{-1}\end{cases}
$$

where $a d-b c=1, a, b, c, d \in L, c x^{1}+d, d-c \xi^{3} \in U(L)$. 
Proof. Identity (22) is solvable with respect to $x^{1 \prime}$, after which we fix the coordinates of the points of the second set. After redefinition, we obtain the first equation of system (23).

Further, the identity (18) we write for sequences $\left\langle\mu_{1} \nu\right\rangle,\left\langle\mu_{2} \nu\right\rangle,\left\langle\mu_{3} \nu\right\rangle$ :

$$
\begin{aligned}
& \left(x^{1 \prime}\left(\mu_{1}\right) \xi^{1 \prime}+\xi^{2 \prime}\right)\left(x^{1 \prime}\left(\mu_{1}\right)+\xi^{3 \prime}\right)^{-1}=\left(x^{1}\left(\mu_{1}\right) \xi^{1}+\xi^{2}\right)\left(x^{1}\left(\mu_{1}\right)+\xi^{3}\right)^{-1}, \\
& \left(x^{1 \prime}\left(\mu_{2}\right) \xi^{1 \prime}+\xi^{2 \prime}\right)\left(x^{1 \prime}\left(\mu_{2}\right)+\xi^{3 \prime}\right)^{-1}=\left(x^{1}\left(\mu_{2}\right) \xi^{1}+\xi^{2}\right)\left(x^{1}\left(\mu_{2}\right)+\xi^{3}\right)^{-1}, \\
& \left(x^{1 \prime}\left(\mu_{3}\right) \xi^{1 \prime}+\xi^{2 \prime}\right)\left(x^{1 \prime}\left(\mu_{3}\right)+\xi^{3 \prime}\right)^{-1}=\left(x^{1}\left(\mu_{3}\right) \xi^{1}+\xi^{2}\right)\left(x^{1}\left(\mu_{3}\right)+\xi^{3}\right)^{-1} .
\end{aligned}
$$

Then the resulting system is resolved with respect to $\xi^{1 \prime}, \xi^{2 \prime}$ and $\xi^{3 \prime}$, whereupon fix the coordinates of the points of the first set and go to the identity (22). As a result, after redefinitions, we get equalities (23).

Obviously, the group of motions (23) is a $3 s$-parametric group, which includes $3 s$ real parameters.

\section{Conclusion}

Complexification by ordinary complex numbers PS of GTS rank $(2,2),(3,3),(4,4)$ and $(5,5)$ are interpreted by Yu.S. Vladimirov in the theory of physical interactions $[10,11]$. They are given the definition of spinors through the PS of GTS rank $(2,2)$, which are used to describe elementary

particles. Complex PS of GTS high ranks are used to describe the fundamental interactions of elementary particles.

In works [12-14], respectively, the complexification affine group and the complexification projective group are investigated as the PS of GTS.

\section{References}

[1] G.G.Mikhailichenko, R.M.Muradov, Physical structures as geometries of two sets, GASU, Gorno-Altaisk, 2008 (in Russian).

[2] G.G.Mikhailichenko, On a problem in the theory of physical structures, Siberian Math. J., 18(1977), no. 6, 1342-1355 (in Russian).

[3] G.G.Mikhailichenko, V.A.Kyrov, Russian Mathematics, 61(2017), no. 7, 15-24.

DOI: $10.3103 /$ S1066369X17070039

[4] G.G.Mikhailichenko, The solution of functional equations in the theory of physical structures, Dokl. Akad. Nauk SSSR 206(1972), no. 5, 1056-1058.

[5] I.L.Kantor, A.C.Solodovnikov, Hypercomplex numbers, Moscow, Nauka, 1973, (in Russian).

[6] G.G.Mikhailichenko, R.M.Muradov, Russian Mathematics, 52(2008), no. 10, 20-24.

DOI: $10.3103 /$ S1066369X08100034

[7] A.I.Kostrikin, Introduction to Algebra, Moscow, Nauka, 1977. (in Russian).

[8] A.I.Maltsev, Fundamentals of linear algebra, Moscow, Nauka, 1973. (in Russian).

[9] F.R.Gantmacher, Theory of matrices, Moscow, Nauka, 1973. (in Russian). 
[10] Yu.I.Kulakov, Yu.S.Vladimirov, A.B.Karnaucnov, Introduction to the theory of physical structures. Moscow, Archimed, 1992. (in Russian).

[11] Yu.S.Vladimirov, The relational theory of space-time. P. 2, Theory of physical interactions. Moscow, MGU, 1999. (in Russian).

[12] V.A.Kyrov, Affine geometry as a physical structure, SibFU Journals. Math and Physics, 1(2008), no. 4, 460-464 (in Russian).

[13] V.A.Kyrov, Projective geometry and phenomenological symmetry, SibFU Journals. Math and Physics, 5(2012), no. 1, 82-90 (in Russian).

[14] V.A.Kyrov, Russian Mathematics, 52(2008), no. 11, 42-52.

DOI: $10.3103 / \mathrm{S} 1066369 \mathrm{X} 08110054$

\title{
Коммутативные гиперкомплексные числа и геометрия двух множеств
}

Владимир А. Кыров

Горно-Алтайский государственный университет Горно-Алтайск, Российская Федерация

\begin{abstract}
Аннотация. Главной задачей теории феноменологически симметричных геометрий двух множеств является классификация таких геометрий. В данной работе по функциям пары точек некоторых известных феноменологически симметричных геометрий двух множеств (ФС ГДМ) с помощью комплексификации ассоциативными гиперкомплексными числами находим функции пары точек новых геометрий. Находим также уравнения групп движений этих геометрий. Устанавливаем феноменологическую симметрию этих геометрий, то есть находим функциональные связи между функциями пары точек для определенного конечного числа произвольных точек. В частности, по однокомпонентным функциям пары точек ФС ГДМ рангов $(\mathrm{n}, \mathrm{n})$ и $(\mathrm{n}+1, \mathrm{n})$ определяем $s+1$ компонентные функции пары точек тех же рангов. Для них находим конечные уравнения групп движений и уравнения, выражающие их феноменологическую симметрию.
\end{abstract}

Ключевые слова: геометрия двух множеств, феноменологическая симметрия, групповая симметрия, гиперкомплексные числа. 